\title{
TOTAL BOUNDEDNESS AND THE AXIOM OF CHOICE
}

\author{
GONÇALO GUTIERRES
}

Dedicated to the memory of Horst Herrlich

\begin{abstract}
A metric space is Totally Bounded (also called preCompact) if it has a finite $\varepsilon$-net for every $\varepsilon>0$ and it is preLindelöf if it has a countable $\varepsilon$-net for every $\varepsilon>0$. Using the Axiom of Countable Choice (CC), one can prove that a metric space is topologically equivalent to a Totally Bounded metric space if and only if it is a preLindelöf space if and only if it is a Lindelöf space.

In the absence of $\mathbf{C C}$, it is not clear anymore what should the definition of preLindelöfness be. There are two distinguished options. One says that a metric space $X$ is:

(a) preLindelöf if, for every $\varepsilon>0$, there is a countable cover of $X$ by open balls of radius $\varepsilon([11])$;

(b) Quasi Totally Bounded if, for every $\varepsilon>0$, there is a countable subset $A$ of $X$ such that the open balls with centers in $A$ and radius $\varepsilon$ cover $X$.

As we will see these two notions are distinct and both can be seen as a good generalization of Total Boundedness. In this paper we investigate the choice-free relations between the classes of preLindelöf spaces and Quasi Totally Bounded spaces, and other related classes, namely the Lindelöf spaces.

Although it follows directly from the definitions that every pseudometric Lindelöf space is preLindelöf, the same is not true for Quasi Totally Bounded spaces. Generalizing results and techniques used by Horst Herrlich in [8], it is proven that every pseudometric Lindelöf space is Quasi Totally Bounded iff Countable Choice holds in general or fails even for families of subsets of $\mathbb{R}$ (Theorem 2.5).
\end{abstract}

\section{INTRODUCTION}

In the last years of his life Horst Herrlich dedicated many of his time studying the impact of the Axiom of Choice in Mathematics and, in particular, in set-theoretic Topology. Of particular interest to Horst was to see how different definitions of the same notion split in the absence of the Axiom, like he did in the pioneer paper Compactness and the Axiom of Choice [7] with different definitions of compactness. Following his steps, I want to give my modest contribution to this subject by looking at the choice-free definition(s) of a preLindelöf space.

The definition of preLindelöf is inspired by the definition of preCompact metric spaces which are more often called Totally Bounded metric spaces. Although both names refer to the same notion, one could say that the term preCompact is more "topological" and that Totally Bounded refers more to the metric. In other words, a metric space $X$ is preCompact if, for every $\varepsilon>0$, it can be covered by finitely many open balls of radius $\varepsilon$; and $X$ is Totally Bounded if, for every $\varepsilon>0$, there is a finite subset $F$ such that for every $x \in X$, exists $a \in F$ such that $d(x, a)<\varepsilon$. There is no doubt that both definitions coincide even in ZF, Zermelo-Fraenkel set theory without the Axiom of Choice. The same is not true when replacing finitely many by countably many. This leads to the definition of the two different classes

2010 Mathematics Subject Classification. 03E25, 54D20, 54E35.

Key words and phrases. Totally Bounded metric space, Countable Choice, Lindelöf space.

This work was partially supported by the Centre for Mathematics of the University of Coimbra - UID/MAT/00324/2013, funded by the Portuguese Government through FCT/MEC and cofunded by the European Regional Development Fund through the Partnership Agreement PT2020. 
(in ZF, as we shall see in Proposition 4.1) of preLindelöf and Quasi Totally Bounded metric spaces, respectively.

This paper was motivated by the definition of preLindelöfness from K. Keremedis in [11]. There, this condition was studied and compared with some other Lindelöf related conditions. The notion of Quasi Total Boundedness was not introduced there and it is my goal in this paper to make more clear the differences between both classes.

It is known that, assuming the Axiom of Countable Choice, a metric space is preLindelöf iff it is Quasi Totally Bounded iff it is Topologically Totally Bounded iff it is Lindelöf iff it is Second Countable iff it is Separable (Proposition 1.6). We will investigate the relations between these notions in the choice-free environment, for metric spaces, pseudometric spaces and subspaces of the real line $\mathbb{R}$. Here, a pseudometric space is a metric space except for the fact that the distance between two distinct points can be 0 . Among these results, I emphasize Theorem 2.5 where is stated that every pseudometric Lindelöf space is Quasi Totally Bounded if the Countable Choice holds or drastically fails. This is a kind of result that Horst enjoyed because it requires two completely independent proofs of the same result. To prove it we make use of one of my favourite results from Horst Herrlich: Lindelöf=Compact for $T_{1}$-spaces is equivalent to the failure of $\mathbf{C C}(\mathbb{R})$ - Theorem 2.1 in $[8]$.

\section{Definitions and Preliminary Results}

We start this section with a list of definitions of set-theoretic axioms which will be used throughout the paper. All results in this paper take place in the setting of ZF.

\section{Definitions 1.1.}

(a) The Axiom of Countable Choice (CC) states that every countable family of non-empty sets has a choice function.

(b) The Countable Union Condition (CUC) states that a countable union of countable sets is countable.

(c) $\mathbf{C C}\left(\aleph_{0}\right)$ is the Axiom of Countable Choice restricted to non-empty families of countable sets.

(d) $\mathbf{C C}(\mathbb{R})$ is the Axiom of Countable Choice restricted to families of non-empty sets of real numbers.

Remark 1.2. Note that $\mathbf{C C} \Rightarrow \mathbf{C U C} \Rightarrow \mathbf{C C}\left(\aleph_{0}\right)$.

Proposition 1.3. ([3, p.76], [9, p.15]) The following conditions are equivalent to $\mathbf{C C}$ (respectively $\mathbf{C C}(\mathbb{R}))$ :

(i) every countable family of non-empty sets (resp. subsets of $\mathbb{R}$ ) has an infinite subfamily with a choice function;

(ii) for every countable family $\left(X_{n}\right)_{n \in \mathbb{N}}$ of non-empty sets (resp. subsets of $\mathbb{R}$ ), there is a sequence which takes values in an infinite number of the sets $X_{n}$.

Condition (i) of the previous proposition is known as the partial Axiom of Countable Choice (PCC).

Given a (pseudo)metric space, an open ball of radius $\varepsilon>0$ in $X$ is a set $B$ such that there exists $x \in X$ with $B=\{y \in X \mid d(x, y)<\varepsilon\}$. We denote an open ball of radius $\varepsilon>0$ and center $x$ by $B_{\varepsilon}(x)$. Clearly, an open ball may have more than one center. This fact motivates us to distinguish between the following two notions, (a) and (b).

Definitions 1.4. Let $(X, d)$ be a (pseudo)metric space. We say that $X$ is: 
(a) preLindelöf (PL) if, for every $\varepsilon>0$, there exists a countable family $\mathcal{A}$ of open balls of radius $\varepsilon$ such that $X=\bigcup \mathcal{A}$;

(b) Quasi Totally Bounded (QTB) if, for every $\varepsilon>0$, there exists a countable set $A \subseteq X$ such that $X=\bigcup_{a \in A} B_{\varepsilon}(a)$;

(c) topologically preLindelöf (TPL) if it is topologically equivalent to a preLindelöf space;

(d) topologically Quasi Totally Bounded (TQTB) if it is topologically equivalent to a Quasi Totally Bounded space;

(e) Totally Bounded (TB) if for every $\varepsilon>0$, there exists a finite set $A \subseteq X$ such that $X=\bigcup_{a \in A} B_{\varepsilon}(a)$;

(f) topologically Totally Bounded (TTB) if it is topologically equivalent to a Totally Bounded space.

Definitions 1.5. Let $(X, d)$ be a (pseudo)metric space and $\mathcal{T}(d)$ the induced topology. We say that $X$ is:

(a) Lindelöf (L) if every cover of $X$ by elements of $\mathcal{T}(d)$ has a countable subcover.

(b) Second Countable (SC) if $(X, \mathcal{T}(d))$ has a countable base.

(c) Separable (S) if it has a countable dense subset.

The fact that, for a (pseudo)metric space, these three definitions are equivalent and equivalent to topologically Total Boundedness in ZFC, Zermelo-Fraenkel set theory with the Axiom of Choice, is well-known. Maybe less known is that they are also equivalent to the definitions (a)-(d) of 1.4. It is surprising that if a metric space can be covered by countably many open balls of radius $\varepsilon$, for every $\varepsilon$, so it can for any other equivalent metric.

Proposition 1.6 (ZF+CC). For a (pseudo)metric space $X$, the following properties are equivalent:

(i) $X$ is Lindelöf;

(ii) $X$ is Second Countable;

(iii) $X$ is Separable;

(iv) $X$ is topologically Totally Bounded;

(v) $X$ is Quasi Totally Bounded;

(vi) $X$ is topologically Quasi Totally Bounded;

(vii) $X$ is preLindelöf;

(viii) $X$ is topologically preLindelöf.

Proof. The equivalences between (i), (ii), (iii) and (iv) can be seen in many introductory books of Topology (e.g. [2]). It is not difficult to see that $\mathbf{C C}$ suffices to prove these equivalences.

It is clear that, under $\mathbf{C C}$, (iv) $\Rightarrow($ vi) $\Leftrightarrow$ (viii) and that (iii) $\Rightarrow(\mathrm{v}) \Leftrightarrow$ (vii) $\Rightarrow$ (viii).

It is now enough to prove that (vi) implies (iii), which is the same as to prove that (v) implies (iii). Let $X$ be a QTB metric space. By definition, for all $n \in \mathbb{N}$, exists a countable set $A_{n} \subseteq X$ such that $X=\bigcup_{a \in A_{n}} B_{1 / n}(a)$. The set $A=\bigcup_{n} A_{n}$ is dense in $X$ and, by the Countable Union Condition, it is countable.

Some of these implications remain valid in ZF. We will write $\mathrm{A} \rightarrow \mathrm{B}$ to say that every space with the property A also has the property B.

Remark 1.7. Most of the equivalent conditions of Proposition 1.6 do not depend on the points, then in these cases it does not matter if one works with metric or with pseudometric spaces. To make it precise, a pseudometric space is preLindelöf (Lindelöf, Second Countable, Totally Bounded) if and only if its metric reflection 
is preLindelöf (Lindelöf, Second Countable, Totally Bounded). Clearly the same is not true for Quasi Total Boundedness or Separability. We recall that the metric reflection is the quotient space resulting from identifying the points for which the distance between them is equal to zero.

The fact that the metric reflection of a Separable (Quasi Totally Bounded) pseudometric space is Separable (Quasi Totally Bounded) is equivalent to the Axiom of Countable Choice. The proof is straightforward and can be done using the pseudometric space defined in Theorem 2.5 below.

Proposition 1.8 (ZF). For (pseudo)metric spaces, the following implications are valid.
(a) $\mathrm{S} \rightarrow \mathrm{SC}$.
(b) $\mathrm{SC} \rightarrow \mathrm{TTB}$.
(c) $\mathrm{TTB} \rightarrow \mathrm{TQTB} \rightarrow$ TPL.
(d) $\mathrm{TB} \rightarrow \mathrm{QTB} \rightarrow \mathrm{PL}$.
(e) $\mathrm{L} \rightarrow \mathrm{PL}$.
(f) $\mathrm{S} \rightarrow$ QTB.

Proof. The usual proof of (a) remains valid in ZF and (c), (d) and (e) are trivial. The fact that every Second Countable metric space is topologically Totally Bounded (b) relies on the fact that a Second Countable metric space can be embedded in $[0,1]^{\aleph_{0}}$. It was pointed out in [4] that this fact holds true in ZF, and this is enough in view of the previous remark. In [1], it is given a detailed proof.

(f) If $(X, d)$ is Separable, then exists $A \subseteq X$ such that $A$ is countable and dense in $X$. Clearly, for every $\varepsilon>0, X=\bigcup_{a \in A} B_{\varepsilon}(a)$.

\section{LINDELÖF SPACES}

In this section we will investigate if the Lindelöf condition is still stronger, in ZF, than the conditions of being preLindelöf or Quasi Totally Bounded.

The implication L $\rightarrow$ PL is quite obvious, but it is not immediate to conclude that $\mathrm{L} \rightarrow$ QTB. Although this last implication is not valid for pseudometric spaces, in ZF, it is valid for metric spaces. We will show these two facts in Theorem 2.5 and Proposition 2.1, respectively.

Proposition 2.1. Every Lindelöf metric space is Quasi Totally Bounded.

Proof. Let $X$ be a Lindelöf metric space. Fix $\varepsilon>0$. Since in a metric space the singleton sets are closed, for every $x \in X$ the sets $B_{\varepsilon}(x) \backslash\{x\}$ are open. Define now $A:=\left\{x \in X \mid(\forall y \in X)\left[(x \neq y) \Rightarrow B_{\varepsilon}(x) \neq B_{\varepsilon}(y)\right]\right\}$.

Consider the open cover of $X$,

$$
\mathcal{U}:=\left\{B_{\varepsilon}(x) \mid x \in A\right\} \cup\left\{B_{\varepsilon}(x) \backslash\{x\} \mid x \notin A\right\} .
$$

Since $y \in B_{\varepsilon}(x)$ if and only if $x \in B_{\varepsilon}(y)$, for all $x, y$ in $X$, we have that $B_{\varepsilon}(x) \neq$ $B_{\varepsilon}(y) \backslash\{y\}$ and that for all $x \neq y$ in $X \backslash A, B_{\varepsilon}(x) \backslash\{x\} \neq B_{\varepsilon}(y) \backslash\{y\}$. For this last statement notice that if $x \notin A$, there is $z \neq x$ such that $B_{\varepsilon}(x)=B_{\varepsilon}(z)$, and thus for every $y \in X, x \in B_{\varepsilon}(y)$ if and only if $z \in B_{\varepsilon}(y)$.

From the above observations we may conclude that every element of $\mathcal{U}$ can only be written in one form.

Since $X$ is Lindelöf, there are $\left(x_{n}\right)_{n}$ in $A$ and $\left(y_{n}\right)_{n}$ in $X \backslash A$ such that

$$
\left\{B_{\varepsilon}\left(x_{n}\right) \mid n \in \mathbb{N}\right\} \cup\left\{B_{\varepsilon}\left(y_{n}\right) \backslash\left\{y_{n}\right\} \mid n \in \mathbb{N}\right\}
$$

is a countable subcover of $\mathcal{U}$. This implies that $X=\bigcup_{n}\left(B_{\varepsilon}\left(x_{n}\right) \cup B_{\varepsilon}\left(y_{n}\right)\right)$ and so $X$ is a Quasi Totally Bounded metric space.

Theorem 2.2. [10] Every Second Countable space is Lindelöf if and only if the Axiom of Countable Choice for subsets of $\mathbb{R}(\mathbf{C C}(\mathbb{R}))$ holds. 
Theorem 2.3. [8] Every Lindelöf $T_{1}$-space is Compact if and only if $\mathbf{C C}(\mathbb{R})$ does not hold.

Corollary 2.4. Every Lindelöf space, for which the $T_{0}$-reflection is $T_{1}$, is Compact if and only if $\mathbf{C C}(\mathbb{R})$ does not hold.

The corollary is straightforward since the $T_{0}$-reflection (the quotient space which identifies the points with he same closure) does not modify the topology. In particular, we have that the failure of $\mathbf{C C}(\mathbb{R})$ implies that every Lindelöf pseudometric space is Compact.

With this last result in place, we are able to proof that, in the pseudometric case, there are Lindelöf spaces which are not Quasi Totally Bounded.

Theorem 2.5. Every Lindelöf pseudometric space is Quasi Totally Bounded if and only if $\mathbf{C C}$ holds or $\mathbf{C C}(\mathbb{R})$ fails.

Proof. $(\Leftarrow)$ If $\mathbf{C C}$ holds, then the usual proof works. If $\mathbf{C C}(\mathbb{R})$ fails every Lindelöf pseudometric space is Compact, hence Totally Bounded and consequently QTB.

$(\Rightarrow)$ Let $\left(X_{n}\right)_{n}$ be a countable family of non-empty sets. Define $X=\bigcup_{n}\left(X_{n} \times\right.$ $\{n\})$ and a pseudometric $d$ on $X$ with

$$
d((x, n),(y, m))=1 \text { if } n \neq m \text {; and } d((x, n),(y, n))=0 .
$$

If $\mathbf{C C}(\mathbb{R})$ holds, then since $(X, d)$ is second countable, it follows, by Theorem 2.2, that $X$ is Lindelöf, and by our hypothesis, $X$ is also QTB. This means that there exists a sequence $\left(x_{k}\right)_{k}$ of points in $X$ such that $X=\bigcup_{k} B_{1 / 2}\left(x_{k}\right)$. Finally, the set $\left\{\pi_{1}\left(x_{k}\right) \mid k \in \mathbb{N}\right\}$, where $\pi_{1}$ is the canonical projection, must intersect all the sets $X_{n}$ which means that $\mathbf{C C}$ also holds. That is $\mathbf{C C}(\mathbb{R})$ implies $\mathbf{C C}$.

The next diagram shows the implications which are known to be true in ZF.

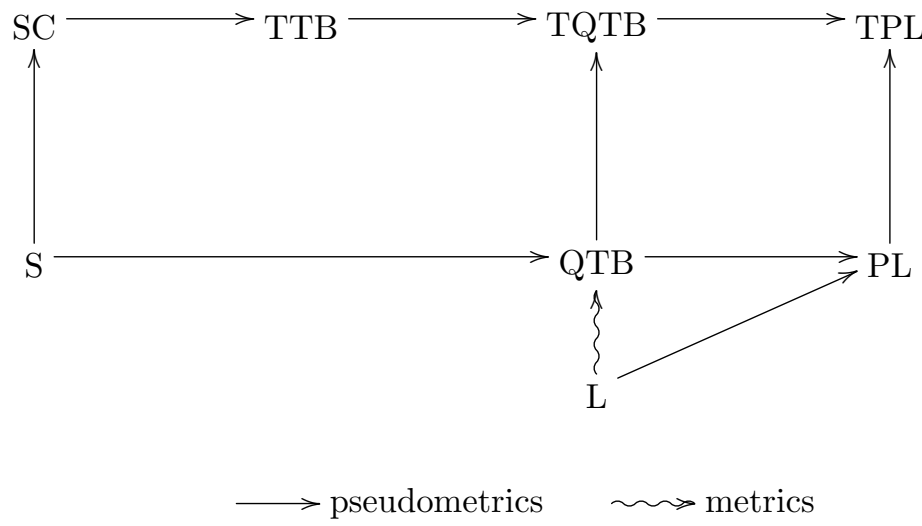

\section{Subspaces of the Reals}

Before studying the set-theoretic status of the implications between preLindelöfness, Quasi Total Boundedness and the other properties for metric spaces, we will see what happens for subspaces of $\mathbb{R}$. Clearly, if an implication is not true for subspaces of $\mathbb{R}$ it is also not true for metric spaces in general.

The metric space $\mathbb{R}$, with the Euclidean metric, is Separable (hence SC, TTB, PL, QTB) and it is Lindelöf if and only if $\mathbf{C C}(\mathbb{R})$ holds (see [10]). So, using Theorem 2.2 , it is clear that the condition $\mathrm{A} \rightarrow \mathrm{L}$ for subspaces of $\mathbb{R}$ is equivalent to $\mathbf{C C}(\mathbb{R})$ with A being any of the other conditions written before.

Before proceeding, we will prove a result that help us showing the Theorem 3.2 below. The technique employed to prove this proposition is also used to prove some 
results in my $\mathrm{PhD}$ Thesis $[6, \mathrm{p} .23]$ and it was suggested by my supervisor Horst Herrlich.

Proposition 3.1. The following conditions are equivalent:

(i) $\mathbf{C C}(\mathbb{R})$, the Axiom of Countable Choice for subsets of $\mathbb{R}$;

(ii) $\mathbf{P C C}(\mathbb{R})$, the partial Axiom of Countable Choice for subsets of $\mathbb{R}$;

(iii) $\mathbf{C C}(d \mathbb{R})$, the Axiom of Countable Choice for dense subspaces of $\mathbb{R}$;

(iv) $\mathbf{P C C}(d \mathbb{R})$, the partial Axiom of Countable Choice for dense subspaces of $\mathbb{R}$.

Proof. The equivalence (i) $\Leftrightarrow($ ii) is in the Proposition 1.3. It is clear that (i) $\Rightarrow$ (iii) $\Rightarrow$ (iv). Now, it is only necessary to prove that (iv) $\Rightarrow$ (ii).

Let $\left(A_{n}\right)_{n}$ be a countable family of non-empty subsets of $\mathbb{R}$. Consider the collection $\left(q_{k}, s_{k}\right)_{k \in \mathbb{N}}$ of all open intervals with rational endpoints and the bijective functions $f_{k}: \mathbb{R} \rightarrow\left(q_{k}, s_{k}\right)$.

Now we define, for every $n$, the set $B_{n}=\bigcup_{k} f_{k}\left(A_{n}\right)$. By the way they were built, each of the sets $B_{n}$ is dense in $\mathbb{R}$. By (iv) there is a sequence $\left(b_{\varphi(n)}\right)_{n}$ with $\varphi$ an increasing sequence of naturals and $b_{\varphi(n)} \in B_{\varphi(n)}$, for every $n \in \mathbb{N}$. We define also $k(n):=\min \left\{k \in \mathbb{N} \mid b_{\varphi(n)} \in f_{k}\left(A_{\varphi(n)}\right)\right\}$. The sequence $\left(a_{\varphi(n)}\right)_{n}$, with $a_{\varphi(n)}:=f_{k(n)}^{-1}\left(b_{\varphi(n)}\right) \in A_{\varphi(n)}$, is the desired partial choice function.

Theorem 3.2. The following conditions are equivalent to $\mathbf{C C}(\mathbb{R})$ :

(i) every dense subspace of $\mathbb{R}$ is Lindelöf;

(ii) every dense subspace of $\mathbb{R}$ is Separable [6];

(iii) every dense subspace of $\mathbb{R}$ is Quasi Totally Bounded;

(iv) every dense subspace of $\mathbb{R}$ is preLindelöf.

Proof. Every subspace of $\mathbb{R}$ is Lindelöf if and only if every subspace of $\mathbb{R}$ is Separable if and only if $\mathbf{C C}(\mathbb{R})$ holds ([10]). Then, it is clear that $\mathbf{C C}(\mathbb{R})$ implies (i) and (ii). From Propositions 1.8 and 2.1, (i) $\Rightarrow$ (iii) and (ii) $\Rightarrow$ (iii) $\Rightarrow$ (iv).

It only remains to prove that (iv) $\Rightarrow \mathbf{C C}(\mathbb{R})$. By Proposition 3.1 , it suffices to prove that (iv) implies the Axiom of Countable Choice for dense subspaces of $\mathbb{R}$. Let $\left(A_{n}\right)_{n}$ be a family of dense subspaces of $\mathbb{R}$. Using the fact that every open interval is homeomorphic to $\mathbb{R}$, one can consider $A_{2 n}$ dense in $(n, n+1)$ and $A_{2 n+1}$ dense in $(-n-1,-n)$. The set $A=\bigcup_{n} A_{n}$ is dense in $\mathbb{R}$. By (iv), there is a countable family $\left(B_{n}\right)_{n}$ such that each $B_{n}$ is an open ball of radius $1 / 2$ and $A=\bigcup_{n} B_{n}$. Since $A$ is dense in $\mathbb{R}$, each open ball has a unique center and so, for every $n \in \mathbb{N}$, there is only one $x_{n}$ such that $B_{n}=B_{1 / 2}\left(x_{n}\right)$. The sequence $\left(x_{n}\right)_{n}$ induces a choice function of $\left(A_{n}\right)_{n}$.

Corollary 3.3. The following conditions are equivalent to $\mathbf{C C}(\mathbb{R})$ :

(i) every subspace of $\mathbb{R}$ is Quasi Totally Bounded;

(ii) every subspace of $\mathbb{R}$ is preLindelöf.

Proposition 3.4. The following conditions are equivalent to $\mathbf{C C}(\mathbb{R})$ :

(i) every preLindelöf subspace of $\mathbb{R}$ is Separable;

(ii) every preLindelöf subspace of $\mathbb{R}$ is Lindelöf;

(iii) every preLindelöf subspace of $\mathbb{R}$ is Quasi Totally Bounded;

(iv) every Quasi Totally Bounded subspace of $\mathbb{R}$ is Separable.

Proof. From the previous results $1.8,2.1$ and 3.2 , we know that $\mathbf{C C}(\mathbb{R}) \Rightarrow(\mathrm{i}) \Rightarrow(\mathrm{iv})$ and that $\mathbf{C C}(\mathbb{R}) \Rightarrow$ (ii) $\Rightarrow$ (iii).

(iii) $\Rightarrow \mathbf{C C}(\mathbb{R})$ Let $\left(A_{n}\right)_{n}$ be a countable family of dense subspaces of $\mathbb{R}$. Without loss of generality, one may consider, for every $n \geq 1$, the set $A_{n}$ to be a dense subspace of $\left(\sum_{k=1}^{n} k, \sum_{k=1}^{n} k+\frac{1}{n}\right)$. 
The set $A=\bigcup_{n} A_{n}$ is preLindelöf. Let $\varepsilon>0$ and let $i \in \mathbb{N}$ be such that $\varepsilon \in[1 / i, i-1]$. For $n>i$, the sets $A_{n}$ are open balls of radius $\varepsilon$ and $\bigcup_{n=1}^{i} A_{n}$ is Totally Bounded because, in $\mathbb{R}$, Total Boundedness coincides with Boundedness. Now, by hypothesis $A$ is also Quasi Totally Bounded which means that there is a sequence $\left(x_{k}\right)_{k}$ in $A$ such that $A=\bigcup_{k} B_{1}\left(x_{k}\right)$. This sequence must take values in each of the sets $A_{n}$, which finishes the proof.

(iv) $\Rightarrow \mathbf{C C}(\mathbb{R})$ Every Bounded subset of $\mathbb{R}$ is Totally Bounded and then Quasi Totally Bounded. So, we have that every bounded subset of $\mathbb{R}$ is Separable. But this last condition it is equivalent to say that every subspace of $\mathbb{R}$ is Separable which is equivalent to $\mathbf{C C}(\mathbb{R})([10])$.

To finish this section, we will address a problem raised in [12, Remark 3.2(B)]. There it is stated that "every Sequentially Compact subspace of $\mathbb{R}$ is preLindelöf" if and only if the Axiom of Countable Choice holds for Sequentially Compact subspaces of $\mathbb{R}(\mathbf{C C}(\mathrm{sc} \mathbb{R}))$, enlarging a result from [5]. Although the result is true, the proof is not correct because what it is really shown is that "every Sequentially Compact subspace of $\mathbb{R}$ is QTB" if and only if $\mathbf{C C}(\mathrm{sc} \mathbb{R})$ holds.

Next, we will fix that proof (Theorem 2.10). For doing so, we need a couple of preliminary results.

\section{Definitions 3.5.}

(a) A topological space is Sequentially Compact if every sequence has a convergent subsequence.

(b) A metric space is Complete if every Cauchy sequence converges.

Lemma 3.6. [5]

(a) Every Sequentially Compact metric space is Complete.

(b) A Bounded subspace of $\mathbb{R}$ is Complete if and only if it is Sequentially Compact.

The previous lemma shows that there is no difference in considering choice for families of Complete or of Sequentially Compact subspaces of $\mathbb{R}$.

Proposition 3.7. The following conditions are equivalent:

(i) the Axiom of (Countable) Choice for Complete (or Sequentially Compact) subspaces of $\mathbb{R}$;

(ii) $\mathbb{R}$ is the only Complete and dense subspace of $\mathbb{R}$;

(iii) the partial Axiom of Countable Choice for Complete (or Sequentially Compact) subspaces of $\mathbb{R}$;

(iv) the partial Axiom of Countable Choice for Complete (=Sequentially Compact) and dense subspaces of $[0,1]$.

Proof. The equivalence between (i) and (ii) is in [5] and between (i) and (iii) is in [12]. It is clear that (iii) implies (iv) and so it is only necessary to be shown that (iv) implies (ii).

Let $A$ be a Complete and dense subspace of $\mathbb{R}$ and $x \in \mathbb{R}$. Define $A_{n}:=A \cap[x-$ $\left.\frac{1}{n}, x+\frac{1}{n}\right], n \geq 1$ and $f_{n}:\left[x-\frac{1}{n}, x+\frac{1}{n}\right] \rightarrow[0,1]$ a bijective continuous function. From the completeness of $A$, one can conclude that each of the spaces $f_{n}\left(A_{n}\right)$ is Sequentially Compact, and they are also dense in $[0,1]$ because $A$ is dense in $\mathbb{R}$. By (iv) there is a partial choice of $\left(f_{n}\left(A_{n}\right)\right)_{n}$ which induces a partial choice of $\left(A_{n}\right)_{n},\left(a_{\varphi(k)}\right)_{k \in \mathbb{N}}$ with $\varphi$ an increasing sequence on natural numbers. The sequence $\left(a_{\varphi(k)}\right)_{k \in \mathbb{N}}$ is a Cauchy sequence and converges to $x$, then $x \in A$. So, we have proved that $A=\mathbb{R}$ which means that $\mathbb{R}$ has no proper subspaces which are Complete and dense.

Theorem 3.8. The following conditions are equivalent: 
(i) the Axiom of (Countable) Choice for Complete subspaces of $\mathbb{R}$;

(ii) every Complete subspace of $\mathbb{R}$ is Separable;

(iii) every Complete subspace of $\mathbb{R}$ is Quasi Totally Bounded;

(iv) every Complete subspace of $\mathbb{R}$ is preLindelöf;

(v) every Sequentially Compact subspace of $\mathbb{R}$ is Quasi Totally Bounded;

(vi) every Sequentially Compact subspace of $\mathbb{R}$ is preLindelöf.

Proof. Every Sequentially Compact metric space is Complete, every Quasi Totally Bounded metric space is preLindelöf and every Separable metric space is Quasi Totally Bounded. Then, (ii) $\Rightarrow($ iii) $\Rightarrow($ iv) $\Rightarrow($ vi) and (iii) $\Rightarrow(\mathrm{v}) \Rightarrow($ vi). The equivalence between (i) and (ii) is in [5].

We will now show that $(\mathrm{vi}) \Rightarrow(\mathrm{i})$. From the previous proposition, it is only necessary to prove the partial Countable Choice for Sequentially Compact and dense subspaces of $[0,1]$. Let $\left(X_{n}\right)_{n}$ be a countable family of Sequentially Compact and dense subspaces of $[0,1]$. Without loss of generality, one can consider $X_{n} \subseteq[n, n+1]$ dense in the respective intervals. Since each of the spaces $X_{n}$ is Sequentially Compact, the space $X=\bigcup_{n} X_{n}$ is Sequentially Compact unless it has an unbounded sequence. If $X$ has an unbounded sequence, then it induces a partial choice function in the family $\left(X_{n}\right)_{n}$. If such a sequence does not exist, $X$ is Sequentially Compact and, by (vi), it is preLindelöf. The preLindelöf property implies that there is $\left(B_{n}\right)_{n}$ such that $X=\bigcup_{n} B_{n}$ where each $B_{n}$ is an open ball of radius $1 / 2$. Since $X$ is dense in $[0,+\infty]$, the centers of the open balls are unique, which induces the desired choice function of $\left(X_{n}\right)_{n}$.

Remark 3.9. In [5] and [12] there are several other conditions equivalent to the ones of Proposition 3.7 and Theorem 3.8.

\section{Metric Spaces}

In the next two sections we will look at the relations between the notions we have been studying, in the realms of the metric spaces and of the pseudometric spaces. We will mainly be interested in the reverse implications of the ones in the diagram on page 5 .

As it was stated in Remark 1.7, results involving only preLindelöf, Lindelöf, Second Countable and Totally Bounded spaces are identical for metric or for pseudometric spaces.

We start with the result which relates the two notions we have looking at more carefully.

Proposition 4.1. If every preLindelöf metric space is Quasi Totally Bounded, then $\mathrm{CC}(\mathbb{R})$ and $\mathbf{C C}\left(\aleph_{0}\right)$.

Proof. The first part follows from Proposition 3.4. For the second part, let $\left(X_{n}\right)_{n}$ be a countable family of non-empty countable sets. Define the metric space $(X, d)$ with $X=\bigcup_{n}\left(X_{n} \times\{n\}\right), d((x, n),(x, n))=0$ and, for $(x, n) \neq(y, m)$,

$$
d((x, n),(y, m)):= \begin{cases}2 & \text { if } n \neq m \\ \frac{1}{n} & \text { if } n=m .\end{cases}
$$

The space $(X, d)$ is preLindelöf. For $\varepsilon>2, X$ is an open ball of radius $\varepsilon$. For $\varepsilon \leq 2$, choose $k$ to be the smallest natural number such that $\frac{1}{k}<\varepsilon$. If $n \geq k$, then $X_{n} \times\{n\}$ is an open ball of radius $\varepsilon$ and if $n<k$ and $x \in X_{n}$, then $\{x\}=B_{\varepsilon}(x)$. Since the finite union of countable sets is countable, $(X, d)$ is preLindelöf.

By our hypothesis, the space $(X, d)$ is QTB, which means that there is a sequence $\left(x_{k}\right)_{k}$ such that $X=\bigcup_{k} B_{1}\left(x_{k}\right)$. This sequence must intersect all of the sets $X_{n}$ which implies that $\left(X_{n}\right)_{n}$ has a choice function.

Proposition 4.2. For metric spaces, each of the following statements is true. 
(a) $\mathrm{TPL} \rightarrow \mathrm{PL} \Rightarrow \mathbf{C C}(\mathbb{R})+\mathbf{C U C}$.

(b) $\mathrm{TQTB} \rightarrow \mathrm{QTB} \Rightarrow \mathbf{C C}(\mathbb{R})+\mathbf{C U C}$.

(c) $\mathrm{QTB} \rightarrow \mathrm{L} \Rightarrow \mathbf{C C}(\mathbb{R})+\mathbf{C U C}$.

(d) $\mathrm{QTB} \rightarrow \mathrm{SC} \Rightarrow$ CUC.

Proof. $(\Rightarrow \mathbf{C C}(\mathbb{R}))$ Every subspace of $\mathbb{R}$ is topologically equivalent to a bounded subspace of $\mathbb{R}$ and so it is topologically Totally Bounded. Corollary 3.3 says that every subspace of $\mathbb{R}$ is PL (or QTB) if and only if $\mathbf{C C}(\mathbb{R})$. It is easily verifiable, after Theorem 3.2, that every bounded subspace of $\mathbb{R}$ is Lindelöf if and only if $\mathbf{C C}(\mathbb{R})$.

$\left(\Rightarrow\right.$ CUC) Define $X=\bigcup_{n}\left(X_{n} \times\{n\}\right)$ and a metric $d$ on $X$ with

$$
d\left((x, n),(y, m)=\max \left\{\frac{1}{n}, \frac{1}{m}\right\} \text { for }(x, n) \neq(y, m) \text {; and } d((x, n),(x, n))=0 .\right.
$$

The space $(X, d)$ is QTB (hence PL) because if one defines $Z_{n}:=\bigcup_{k \leq n} X_{k} \times\{k\}$, for $\varepsilon>\frac{1}{n}, X=\bigcup_{z \in Z_{n}} B_{\varepsilon}(z)$ and $Z_{n}$ is countable.

(a) and (b). Let $d^{\prime}$ be the discrete metric on $X$. It is not hard to see that both $d$ and $d^{\prime}$ induce the discrete topology on $X$. This means that $\left(X, d^{\prime}\right)$ is TQTB and TPL. From our assumptions, $(X, d)$ is PL. The open balls of radius 1 for the metric $d^{\prime}$ are the singleton sets. Since countably many open balls of radius 1 cover $X, X$ must be countable itself.

(c) and (d). The metric space $(X, d)$ is QTB and $X$ has the discrete topology. A discrete space can only be Lindelöf or Second Countable if it is countable, and so $X$ is countable as we want to prove.

In a similar way of the part (d) of the last proposition, can be proven that the implication $\mathrm{TB} \rightarrow \mathrm{SC}$ is not a theorem of $\mathrm{ZF}$.

Taking into consideration that $\mathrm{S} \rightarrow \mathrm{SC}$ and that QTB $\rightarrow$ PL, the next corollary is straightforward after Propositions 3.4 and 4.2.

Corollary 4.3. For metric spaces, each of the following statements is true.
(a) $\mathrm{PL} \rightarrow \mathrm{SC} \Rightarrow$ CUC.
(b) $\mathrm{QTB} \rightarrow \mathrm{S} \Rightarrow \mathrm{CC}(\mathbb{R})+\mathrm{CUC}$.
(c) $\mathrm{PL} \rightarrow \mathrm{S} \Rightarrow \mathrm{CC}(\mathbb{R})+\mathrm{CUC}$.
(d) $\mathrm{PL} \rightarrow \mathrm{L} \Rightarrow \mathbf{C C}(\mathbb{R})+\mathbf{C U C}$.

Theorem 4.4. For metric spaces, the following implications are equivalent to $\mathbf{C C}(\mathbb{R})$ :
(i) $\mathrm{SC} \rightarrow \mathrm{S}$;
(ii) $\mathrm{SC} \rightarrow \mathrm{QTB}$;
(iii) $\mathrm{SC} \rightarrow \mathrm{PL}$.

Proof. From Theorem 3.8, one has that $\mathbf{C C}(\mathbb{R})$ is equivalent to (i) (see also [11]). Since $\mathrm{S} \rightarrow$ QTB $\rightarrow$ PL - Proposition 1.8- (i) $\Rightarrow$ (ii) $\Rightarrow($ iii). Every subspace of $\mathbb{R}$ is Second Countable and every subspace of $\mathbb{R}$ is preLindelöf if and only if $\operatorname{CC}(\mathbb{R})$ holds - Corollary 3.3. These two facts yeld that (iii) implies $\mathbf{C C}(\mathbb{R})$.

We finish this section by showing two equivalences between some of the implications we have seen are not provable in ZF, following what it was done in [11].

\section{Proposition 4.5 .}

(a) For metric spaces, if $P L \rightarrow L$ then $P L \rightarrow S$.

(b) For (pseudo)metric spaces, if $Q T B \rightarrow L$ then $Q T B \rightarrow S$.

The result of (a) is stated in [11, Theorem 12(ii)], but the proof presented there is incorrect. We will follow that proof until the point where some form of choice was used (in particular, choosing centers from open balls). To be easier for the reader, a complete proof is presented. 
Proof. We will only prove (a), because the proof of (b) is similar and easier.

Let $(X, d)$ be a preLindelöf metric space. One can consider the metric bounded by 1. Define $Y=X \times \mathbb{N}$ and a metric in $Y$

$$
d^{\prime}((x, n),(y, m)):=\left\{\begin{array}{cl}
\frac{1}{n} d(x, y) & \text { if } n=m \\
\max \left\{\frac{1}{n}, \frac{1}{m}\right\} & \text { if } n \neq m .
\end{array}\right.
$$

We will prove now that with this metric $Y$ is preLindelöf. Let $\varepsilon>0$ and let $n \in \mathbb{N}$ be such that $\frac{1}{n}<\varepsilon \leq \frac{1}{n+1}$. Then $B_{\varepsilon}((x, n))=\bigcup_{k \geq n}(X \times\{k\})$ for any $x \in X$. Since $X$ is preLindelöf, for all $k<n$, there exists a countable cover $\mathcal{A}_{k}$ of $X$ by open balls of radius $k \varepsilon$. The set $\left\{A \times\{k\} \mid A \in \mathcal{A}_{k}, k<n\right\} \cup\left\{\bigcup_{k \geq n}(X \times\{k\})\right\}$ is a countable cover by open balls of radius $\varepsilon$. Then, by hypothesis, $Y$ is also Lindelöf.

Define the sets

$$
X_{n}:=\left\{x \in X \mid(\forall y \in X)\left[(x \neq y) \Rightarrow B_{1 / 2^{n}}((x, n)) \neq B_{1 / 2^{n}}((y, n))\right]\right\},
$$

and consider the open cover $\mathcal{U}=\bigcup_{n} \mathcal{U}_{n}$ of $Y$ with

$$
\mathcal{U}_{n}=\left\{B_{1 / 2^{n}}(x, n) \mid x \in X_{n}\right\} \cup\left\{B_{1 / 2^{n}}(x, n) \backslash\{(x, n)\} \mid x \notin X_{n}\right\} .
$$

Since $Y$ is Lindelöf, $\mathcal{U}$ has a countable subcover $\mathcal{V}$. As it was done in the proof of the Proposition 2.1, one can show that each element of $\mathcal{V}$ determines uniquely either its center or the element missing for the set to be an open ball of radius $1 / 2^{n}$. So, the set

$C=\left\{x \in X \mid(\exists n \in \mathbb{N}) B_{1 / 2^{n}}((x, n)) \in \mathcal{V} \cap \mathcal{U}_{n}\right.$ or $\left.B_{1 / 2^{n}}((x, n)) \backslash\{(x, n)\} \in \mathcal{V} \cap \mathcal{U}_{n}\right\}$ is countable.

To complete the proof, it is only necessary to see that $C$ is dense in $X$. Let $x \in X$. For every $n$, there exists $y \in C$ such that $d^{\prime}((x, n),(y, n))<1 / 2^{n} \Leftrightarrow d(x, y)<n / 2^{n}$, which implies that $C$ is dense in $X$ because the sequence $n / 2^{n}$ converges to 0 .

\section{Pseudometric Spaces}

Unlike with metric spaces, the situation of the equivalence between preLindelöfness and QTBoundedness is more clear for pseudometric spaces. We start with the result concerning that equivalence.

Theorem 5.1. Every preLindelöf pseudometric space is Quasi Totally Bounded if and only if the Axiom of Countable Choice holds.

Proof. That Countable Choice suffices to prove the equivalence between PL and QTB, one has already seen in Proposition 1.6.

Let $\left(X_{n}\right)_{n}$ be a countable family of non-empty sets. Define $X=\bigcup_{n}\left(X_{n} \times\{n\}\right)$ and a pseudometric $d$ on $X$ with

$$
d((x, n),(y, m))=1 \text { if } n \neq m \text {; and } d((x, n),(y, n))=0 .
$$

Clearly $(X, d)$ is preLindelöf. If $(X, d)$ is also Quasi Totally Bounded, then there is a sequence $\left(x_{k}\right)_{k}$ in $X$ such that $X=\bigcup_{k} B_{1}\left(x_{k}\right)$. This sequence gives a choice function of $\left(X_{n}\right)_{n}$.

Proposition 5.2. For pseudometric spaces, the following conditions are equivalent to $\mathbf{C C}$ :

(i) every Topologically (Quasi) Totally Bounded space is Quasi Totally Bounded;

(ii) every Second Countable space is Quasi Totally Bounded.

Proof. From Proposition 1.6, $\mathrm{CC} \Rightarrow$ (i) and (i) $\Rightarrow$ (ii) because $\mathrm{SC} \rightarrow \mathrm{TTB} \rightarrow \mathrm{TQTB}$ (Proposition 1.8).

It is only necessary to prove that (ii) implies CC. The space $(X, d)$ defined in the proof of Theorem 5.1 is Second Countable and it is Quasi Totally Bounded if and only if $\mathbf{C C}$ is valid. 
Proposition 5.3. Every (Quasi) Totally Bounded pseudometric space is Separable if and only if the Axiom of Countable Choice holds.

Proof. By Proposition 1.6, Countable Choice suffices to prove the equivalence between QTB and S.

Let $\left(X_{n}\right)_{n}$ be a countable family of non-empty sets. Define $X=\bigcup_{n}\left(X_{n} \times\{n\}\right)$, and a pseudometric $d$ on $X$ with

$$
d((x, n),(y, m))=\max \left\{\frac{1}{n}, \frac{1}{m}\right\} \text { if } n \neq m \text {; and } d((x, n),(y, m))=0 \text { if } n=m .
$$

We will see that $(X, d)$ is Totally Bounded. Let $\varepsilon>0$ and $n \in \mathbb{N}$ be such that $\varepsilon>\frac{1}{n}$. By finite choice, one has a finite sequence $\left(x_{k}\right)_{k \leq n}$ with $x_{k} \in X_{k}$. Then $X=\bigcup_{k \leq n} B_{\varepsilon}\left(\left(x_{k}, k\right)\right)$. By our hypothesis, $X$ is also separable which means that there is a sequence $\left(z_{n}\right)_{n}$ dense in $X$. Then $\left(z_{n}\right)_{n}$ takes values in each $X_{n}$, hence $\left(X_{n}\right)_{n}$ has a choice function.

We finish this paper enlarging the implication diagram of page 5 to show the set-theoretic status, for pseudometric spaces, of the reverse implications of that diagram.

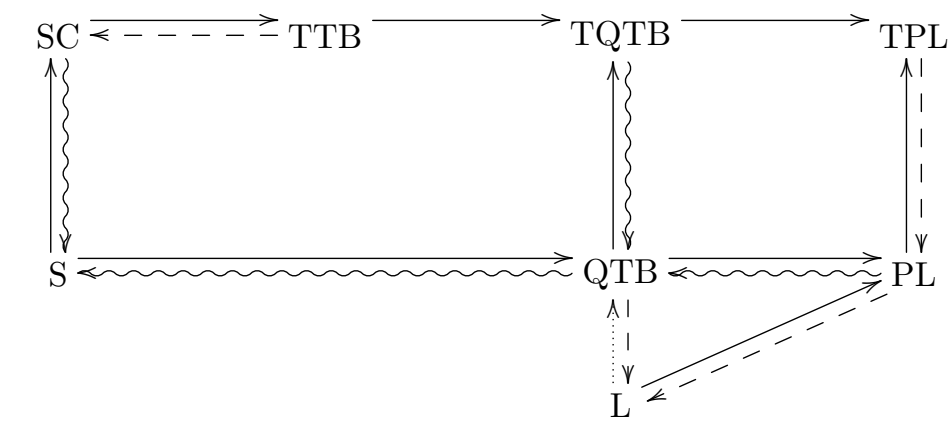

$\longrightarrow \mathrm{ZF} \leadsto \mathbf{C C} \quad \sim->$ not true in ZF $\quad \ldots \ldots \ldots . . . \mathrm{CC}$ or not $\mathbf{C C}(\mathbb{R})$

\section{REFERENCES}

1. H. L. Bentley and H. Herrlich, Countable choice and pseudometric spaces, Topology Appl. 85 (1997), 153-164.

2. R. Engelking, General Topology, revised and completed edition, Sigma Series in Pure Mathematics, vol. 6, Heldermann Verlag, Berlin, 1989.

3. A. A. Fraenkel, Y. Bar-Hillel, and A. Levy, Foundations of set theory, Second revised edition, North-Holland Publ. Co., Amsterdam London, 1973.

4. C. Good and I. J. Tree, Continuing horrors in topology without choice, Topology Appl. 63 (1995), 79-90.

5. G. Gutierres, Sequential topological conditions in $\mathbb{R}$ in the absence of the axiom of choice, Math. Log. Quart. 49 (2003), 293-298.

6. G. Gutierres, O Axioma da Escolha Numerável em Topologia, Ph.D. thesis, Universidade de Coimbra, 2004. (www.mat.uc.pt/ ggutc)

7. H. Herrlich, Compactness and the Axiom of Choice, Appl. Categ. Structures 4 (1996), 1-14.

8. H. Herrlich, Products of Lindelöf $T_{2}$-spaces are Lindelöf - in some models of ZF, Comment. Math. Univ. Carolinae 43 (2002), 319-333.

9. H. Herrlich, Axiom of Choice, Lecture Notes in Mathematics 1876, Springer, Heidelberg, 2007.

10. H. Herrlich and G. E. Strecker, When is $\mathbb{N}$ Lindelöf?, Comment. Math. Univ. Carolinae 38 (1997), 553-556.

11. K. Keremedis, The failure of the axiom of choice implies unrest in the theory of Lindelöf metric spaces, Math. Log. Quart. 49 (2003) 179-186.

12. K. Keremedis and E. Tachtsis, On sequentially compact subspaces of $\mathbb{R}$ without the axiom of choice, Notre Dame J. Formal Logic 44 (2003) 175-184. 
CMUC, Department of Mathematics, University of Coimbra, 3001-454 Coimbra, PorTUGAL

E-mail address: ggutc@mat.uc.pt 